\title{
HS10296, a novel irreversible EGFR TKI, attenuates epithelial-mesenchymal transition facilitated by M2-polarizedtumor-associated macrophages in Non- Small Cell Lung Cancer
}

\author{
Fang Wei, Jing Zhou, Zhi Rui Zhang, Hao Liu \\ School of Pharmacy, vice director of the department of pharmacology, China
}

Background: Many studies have linked the EMT to the emergence of epidermal growth factor receptor-tyrosine kinase inhibitors (EGFR-TKIs) resistance in lung cancer. However, the underlying mechanisms remain to be fully elucidated. M2-polarized macrophages play a pivotal role in tumor microenvironment. In this study, we explore whether HS10296, a novel irreversible EGFR-TKI, restrain EMT of lung cancer cell by suppressing M2-polarized macrophages.

Methods:We conducted a relationship analysis between M2-polarized TAMs infiltration and EMT in NSCLC patients. The effects of HS10296 in vivo were clarified with xenografts mouse. The mechanism of HS10296 was investigated in M2-TAMs co-cultured with A549 and H1975 cells in vitro.

Results: We found that the expression of CD163 was strong positive correlation between the expression of vimentin $(\mathrm{P}<0.001)$, while the expression of CD163 was negatively correlated with the expression of E-cadherin $(\mathrm{P}<0.001)$. M2polarized TAMs promote proliferation, migration and invasion of NSCLC cells, which were distinctly inhibited by treatment with HS10296. HS10296 reduced the number of M2-TAMs and decreased M2-TAMs induced the expression of vimentin, p-AKT, p-STAT3, p-ERK in NSCLC cells. Furthermore, we identify the effects of HS10296 in vivo, HS10296 administration inhibited the volume and weight of tumor significantly in subcutaneous xenografts mouse, reduced expression of CD68/Arginase I obviously. HS10296 lessen macrophage recruitment and inhibited EMT at the tumor site by down-regulation of EGFR pathway.

Conclusions:The results showed that M2-polarized TAMs was associated with EMT which play an important role in the proliferation, migration, invasion and chemo-resistance of NSCLC cells. Moreover, the antitumor mechanism of HS10296 is related to interruption of the link between TAMs and cancer cells by inhibiting EGFR pathway in vitro and in vivo. Our results demonstrated HS10296 is a novel potential TKI for NSCLC. 